\title{
Comparison of Limus-Eluting and Paclitaxel-Eluting Stents for Coronary Intervention in Patients with Chronic Kidney Disease A Meta-Analysis
}

\author{
Xiuhui Yang, ${ }^{1}$ MD, Qingquan Liu, ${ }^{1} \mathrm{PhD}$, Lijun Yuan, ${ }^{1} \mathrm{MD}$, Haoran Wang, ${ }^{1} \mathrm{MD}$ and Qiaotao Xie, ${ }^{1} \mathrm{MD}$
}

\begin{abstract}
Summary
Chronic kidney disease (CKD) patients have worse adverse cardiovascular outcomes after percutaneous coronary intervention (PCI). Clinical outcomes comparing a limus-eluting stent (LES) to a paclitaxel-eluting stent (PES) in patients with CKD remain controversial.

A systematic search was conducted using PubMed, EMBASE, and Cochrane Library. A pooled odds ratio (OR) and 95\% confidence interval (CI) were used to calculate original data. We conducted heterogeneity, quality assessment, and publication bias analyses.

A total of 17 trials involving 10,724 patients were included. No significant differences were found regarding target vessel revascularization, target lesion revascularization (TLR), stent thrombosis (ST), myocardial infarction (MI), all-cause mortality, and major adverse cardiac events (MACE) between first-generation LES implantation and PES implantation. Second-generation LES implantation was associated with lower rates of allcause mortality (OR, 0.56; 95\% CI, 0.39-0.82; $P=0.003$ ), MACE (OR, 0.61; 95\% CI, 0.38-0.97; $P=0.04$ ), and ST $(\mathrm{OR}, 0.45 ; 95 \% \mathrm{CI}, 0.26-0.77 ; P=0.004)$ compared with PES implantation. In all, the long-term allcause mortality rate was significantly lower after LES implantation than after PES implantation in patients with CKD (OR, 0.78; 95\% CI, 0.66-0.93; $P=0.004)$. However, second-generation LES implantation resulted in a higher rate of TLR (OR, 2.23; 95\% CI, 1.53-3.25; $P<0.001)$ than PES implantation in dialysis patients.

In patients with CKD, first-generation LES and PES implantation had comparable mortality and morbidity. Second-generation LES implantation was superior to PES in reducing long-term mortality, MACE, and ST. However, PES may be more effective than LES in dialysis patients.
\end{abstract}

Key words: Percutaneous coronary intervention, Outcomes

(Int Heart J 2018; 59: 1202-1210)

$\mathrm{R}$ enal insufficiency has been independently associated with increased risk of cardiovascular morbidity and mortality in patients with coronary artery disease. ${ }^{1,2)}$ Moreover, after percutaneous coronary intervention (PCI), patients with chronic kidney disease (CKD) have higher rates of adverse outcomes than patients without CKD. ${ }^{3-5)}$ While performing PCI in these patients using first-generation drug-eluting stents (DES), paclitaxel-eluting stents (PES) and sirolimus-eluting stents (SES) have been reported to reduce restenosis or mortality when compared with bare metal stents (BMS).$^{6-8)}$ Although everolimus-eluting stents (EES), zotarolimus-eluting stents (ZES), and sirolimus-eluting stents (SES) are limuseluting stents (LES) that share similar underlying pharmacological mechanisms, different effects on clinical outcomes after LES versus PES implantation may result from differences in drugs, pharmacokinetics, and polymers.

Previous studies have shown conflicting results in terms of major cardiac adverse events comparing SES to PES in patients with chronic kidney disease. ${ }^{9-12)}$ Newer- generation DES, such as ZES, showed better preserved endothelial-dependent vasomotion and biocompatibility compared to first-generation DES. ${ }^{13,14)}$ However, even if a second-generation DES is expected to be associated with better clinical outcomes in patients with $\mathrm{CKD}$, data regarding the long-term adverse clinical outcomes treated with either EES/ZES or PES are still controversial. ${ }^{15-17)}$

To the best of our knowledge, no meta-analysis thus far has been attempted to investigate whether limuseluting stents or newer-generation DES reduce the risk of mortality and morbidity in patients with CKD compared with PES. Therefore, we performed such a meta-analysis with the aim of comparing clinical events of SES versus PES implantation, or of EES/ZES versus PES implantation in patients with CKD.

\section{Methods}

Literature search: This meta-analysis was performed and reported according to the PRISMA guidelines. ${ }^{18)}$ We con-

From the ${ }^{1}$ Department of Hypertension, Luohe Central Hospital, The First Affiliated Hospital of Luohe Medical College, Henan, China.

Address for correspondence: Qiaotao Xie, MD, Department of Hypertension, Luohe Central Hospital, The First Affiliated Hospital of Luohe Medical College, People's Road 54, Henan, 462000, China. E-mail: xieqt2000@163.com

Received for publication October 11, 2017. Revised and accepted January 15, 2018

Released in advance online on J-STAGE October 25, 2018.

doi: 10.1536/ihj.17-583

All rights reserved by the International Heart Journal Association. 
ducted a search of PubMed, EMBASE and Cochrane Library databases from their date of inception up to March 2017, using key words and/or medical subject heading terms: "sirolimus or everolimus or zotarolimus-eluting stent", "paclitaxel-eluting stent", "percutaneous coronary intervention", "chronic kidney disease", "chronic renal failure" and "dialysis". No language or publication date restrictions were imposed. We also searched the reference lists of all retrieved articles to identify additional eligible studies.

Study inclusion and exclusion criteria: Studies were considered eligible for inclusion if the following criteria were met: (1) randomized controlled trials (RCTs) or observational trials, (2) comparisons of SES versus PES implantation, or of second generation LES (EES or ZES) versus PES implantation in patients with CKD undergoing PCI, and (3) at least 1 outcome as follows: major cardiovascular events (MACEs), all-cause mortality, myocardial infarction (MI), target lesion revascularization (TLR), target vessel revascularization (TVR), and stent thrombosis (ST, including definite and probable) should be reported. Exclusion criteria were (1) animal studies, reviews, case reports, letters, and conferences and (2) a follow-up period of less than 6 months. Two independent investigators (XY and QL) screened the titles and abstracts of all electronic citations. Any inconsistency was settled by discussion with a senior author (QX).

Data extraction and quality assessment: Two investigators (QL and XY) independently reviewed titles and abstracts generated by the search, and the third investigator adjudicated any discrepancies. Data was collected, including the outcomes of interest, general characteristics of the included studies, and study participants. CKD was defined as an estimated glomerular filtration rate (eGFR) of $<60$ $\mathrm{mL} /$ minute $/ 1.73 \mathrm{~m}^{2}$. A duration of follow-up of more than one year was regarded as the long-term outcome. Outcomes were assessed at maximum available follow-up. Our primary outcome was long-term all-cause mortality. Secondary outcomes were major cardiovascular events, myocardial infarction, target lesion revascularization, target vessel revascularization, and stent thrombosis.

The methodological quality of the studies was assessed by means of the Jadad scale for quality of randomized controlled studies $(\mathrm{RCTs})^{19)}$ and the 9-item Newcastle-Ottawa Quality scale ${ }^{20)}$ for quality of observational studies in our meta-analysis. In the current study, we considered a study awarded a Jadad score of $\geq 2$, or a Newcastle-Ottawa score of $\geq 6$ as a high-quality study.

Statistical analysis: Summary OR estimates and 95\% CI (CIs) were calculated for the outcomes of interest. The fixed-effect model was used to calculate pooled ORs among studies. When statistically significant heterogeneity existed, a random-effect model (DerSimonian and Laird) was used to combine the results. ${ }^{21)}$ Statistical heterogeneity was assessed using Cochran's Q test and $\mathrm{I}^{2}$ statistic and a value of $>50 \%$ was considered to indicate significant heterogeneity among studies. Sensitivity analysis by removing one study at a time to evaluate that study's effects on the overall results was performed. Subgroup analyses were conducted according to the type of PCI: SES versus PES implantation or second generation LES (EES or ZES) ver- sus PES. We also carried out subgroup analysis for each end point restricted to patients on chronic dialysis to compare the efficacy and safety of LES versus PES. Publication bias was assessed using a funnel plot and Egger's regression test for the outcome of all-cause mortality. ${ }^{22)}$ If publication bias exists, the funnel plot is asymmetric or the Egger's test $P$ value is $<0.05$. All statistical analyses were carried out with RevMan 5.3 and Stata 12.0 software. We considered a $P$ value $<0.05$ as significant.

\section{Results}

Study selection: The study selection process is shown in Figure 1. Of the 983 articles retrieved during the initial search, 647 were excluded at the title/abstract level. After full-text review of the remaining 98 articles, we excluded 12 articles that were reviews, letters, case reports, or abstracts, 28 articles that did not have comparison groups between limus-eluting and paclitaxel-eluting stents, 14 articles that did not report adverse clinical outcomes, and 27 articles that did not enroll patients with CKD. Finally, a total of 17 studies including 10,724 patients that met our predefined inclusion criteria were included in this study. ${ }^{\text {9-12,15-17,23-32) }}$

Study characteristics and quality assessment: The characteristics and overall quality of the individual trials are shown in Table I. Of these 17 studies, 15 were observational retrospective studies ${ }^{9,12,15-17,23-32)}$ and 2 were randomized controlled studies. ${ }^{10,11)}$ The studies varied in sample size from 68 to 3,201 patients with a follow-up period ranging from 12 months to 47 months. Of these studies, 3 were conducted in the United States, 2 in European countries ( 1 in Netherlands and 1 in Switzerland), and 12 in Asian countries ( 8 in Japan and 4 in Korea). Most studies compared the clinical outcomes between SES and PES, while 5 investigated the clinical outcomes of secondgeneration drug eluting stents (ZES or EES) versus PES in CKD patients. The patients included were predominately male and their mean ages ranged from 64 to 76 years. Seven out of 17 studies evaluated the efficacy and safety of limus-eluting versus paclitaxel-eluting stents for patients with end-stage renal disease on dialysis. ${ }^{11,16,24-26,28,29)}$ In addition, all studies included in our meta-analysis were considered as high quality (RCT with a Jadad score of $\geq$ 2 and observational studies with a Newcastle-Ottawa Quality Assessment Scale score of $\geq 6$ ). The clinical characteristics of the included patients are shown in Table II.

Long-term all-cause mortality: A total of 12 studies (2,416 patients) reported long-term all-cause mortality. The long-term ( $>1$ year) all-cause mortality was significantly lower after LES implantation than after PES implantation (OR, 0.78; 95\% CI, 0.66-0.93; $P=0.004$ ), with low heterogeneity $\left(\mathrm{I}^{2}=8 \%\right.$ ) (Figure 2$)$. In the subgroup analyses (Table III) by type of coronary stent, compared with PES, only secondary generation DES (SES or ZES) was associated with a significant reduction in risk for long-term all-cause mortality (OR, 0.56; 95\% CI, 0.39$\left.0.82 ; P=0.003 ; \mathrm{I}^{2}=18 \%\right)$.

MACE: Fifteen studies $(9,884$ patients $)$ reported the MACE rate after coronary stent implantation. While PES (16.2\%) had a higher MACE rate as compared to LES 


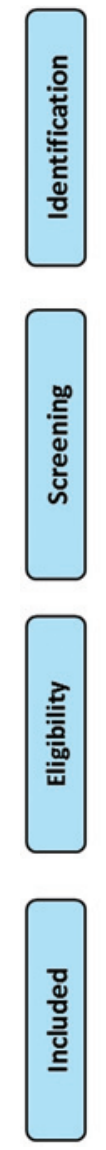

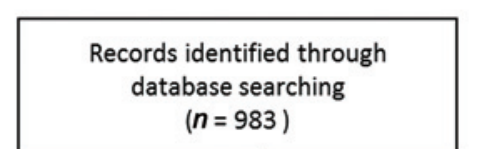

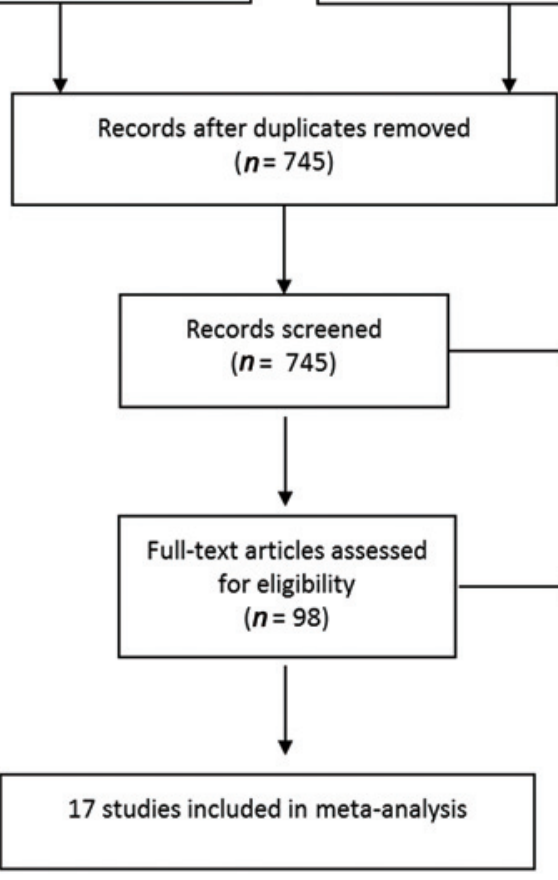

Full-text articles excluded, because they were:

1. Reviews, letters, case reports, abstracts $(\boldsymbol{n}=12)$

2. No comparison groups between limus-eluting and paclitaxel-eluting stents $(n=28)$

3. Adverse clinical outcomes were not reported $(n=14)$

4. Not all in patients with CKD $(n=27)$

Figure 1. Flow diagram of selection process.

Table I. Key Characteristics of Included Studies on LES Versus PES in Patients with Chronic Kidney Disease

\begin{tabular}{|c|c|c|c|c|c|c|c|c|}
\hline \multirow{2}{*}{ Study } & \multirow{2}{*}{ Year } & \multirow{2}{*}{ Country } & \multirow{2}{*}{ Study design } & \multirow{2}{*}{ Stents } & \multicolumn{2}{|c|}{ Patients } & \multirow{2}{*}{ Follow-up } & \multirow{2}{*}{$\begin{array}{c}\text { Quality of } \\
\text { study }\end{array}$} \\
\hline & & & & & LES & PES & & \\
\hline Crimi $^{15)}$ & 2016 & Switzerland & Post-hoc analysis of RCT & EES versus PES & 87 & 98 & 24 months & N9 \\
\hline Crimi $^{15)}$ & 2016 & Switzerland & Post-hoc analysis of RCT & ZES versus PES & 94 & 98 & 24 months & N9 \\
\hline Ann ${ }^{9)}$ & 2016 & Korea & Prospective cohort & SES versus PES & 351 & 351 & 26 months & N9 \\
\hline Kitasato $^{23)}$ & 2015 & Japan & Retrospective cohort & EES versus PES & 73 & 74 & 36 months & N8 \\
\hline Otsuka $^{16)}$ & 2015 & Japan & Retrospective cohort & EES versus PES & 102 & 107 & 12 months & ${ }^{\mathrm{N}} 8$ \\
\hline Otsuka $^{10)}$ & 2014 & Japan & $\mathrm{RCT}$ & SES versus PES & 613 & 593 & 12 months & $\mathrm{J}_{3}$ \\
\hline Sardi $^{24)}$ & 2014 & USA & Prospective cohort & SES or EES versus PES & 156 & 62 & 24 months & $\mathrm{N}_{9}$ \\
\hline Kamoi $^{11)}$ & 2013 & Japan & RCT & SES versus PES & 50 & 50 & 12 months & ${ }^{\mathrm{J}} 5$ \\
\hline Tsujita ${ }^{26)}$ & 2013 & Japan & Retrospective cohort & SES versus PES & 41 & 38 & 12 months & N8 \\
\hline Yano ${ }^{25)}$ & 2013 & Japan & Retrospective cohort & SES versus PES & 32 & 36 & 12 months & ${ }^{\mathrm{N}} 6$ \\
\hline Hachinohe $^{17)}$ & 2012 & Korea & Prospective cohort & SES versus PES & 1845 & 1356 & 12 months & ${ }^{\mathrm{N}} 8$ \\
\hline Hachinohe ${ }^{17)}$ & 2012 & Korea & Prospective cohort & ZES versus PES & 365 & 1356 & 12 months & N8 \\
\hline Simsek $^{12)}$ & 2012 & Netherlands & Retrospective cohort & SES versus PES & 290 & 260 & 72 months & ${ }^{\mathrm{N}} 8$ \\
\hline Ahmed $^{27)}$ & 2012 & Korea & Prospective cohort & SES versus PES & 420 & 381 & 12 months & ${ }^{\mathrm{N}} 6$ \\
\hline Ahmed ${ }^{27)}$ & 2012 & Korea & Prospective cohort & ZES versus PES & 713 & 381 & 12 months & ${ }^{\mathrm{N}} 6$ \\
\hline Ahmed ${ }^{27)}$ & 2012 & Korea & Prospective cohort & EES versus PES & 602 & 381 & 12 months & ${ }^{\mathrm{N}} 6$ \\
\hline Higashitani ${ }^{29)}$ & 2011 & Japan & Retrospective cohort & SES versus PES & 49 & 54 & 12 months & ${ }^{\mathrm{N}} 8$ \\
\hline Otsuka ${ }^{28)}$ & 2011 & Japan & Retrospective cohort & SES versus PES & 184 & 95 & 12 months & N7 \\
\hline Syed ${ }^{31)}$ & 2010 & USA & Retrospective cohort & SES versus PES & 346 & 224 & 12 months & ${ }^{\mathrm{N}} 8$ \\
\hline Sukhija $^{30)}$ & 2010 & USA & Retrospective cohort & SES versus PES & 141 & 287 & 47 months & ${ }^{\mathrm{N}} 8$ \\
\hline Jeong ${ }^{32)}$ & 2007 & Korea & Retrospective cohort & SES versus PES & 76 & 28 & 12 months & N7 \\
\hline
\end{tabular}

SES indicates sirolimus-eluting stent; EES, everolimus-eluting stent; ZES, zotarolimus-eluting stent; LES, limus-eluting stent; PES, paclitaxel-eluting stent; CI, confidence interval; RCT, randomized controlled trials; ', Jadad quality-scoring scale for RCT, maximum score of 5; and ${ }^{\mathrm{N}}$, Newcastle-Ottawa Quality Assessment Scale for non-RCT, maximum score of 9. 
Table II. Key Clinical Characteristics of Included Patients

\begin{tabular}{|c|c|c|c|c|c|c|c|c|c|c|c|c|c|c|}
\hline \multirow[t]{2}{*}{ Study } & \multicolumn{2}{|c|}{$\begin{array}{l}\text { Mean age } \\
\text { (years) }\end{array}$} & \multicolumn{2}{|c|}{ Males (\%) } & \multicolumn{2}{|c|}{$\begin{array}{l}\text { Hypertension } \\
(\%)\end{array}$} & \multicolumn{2}{|c|}{$\begin{array}{c}\text { Diabetes } \\
\text { mellitus } \\
(\%)\end{array}$} & \multicolumn{2}{|c|}{$\begin{array}{c}\text { Prior PCI } \\
(\%)\end{array}$} & \multicolumn{2}{|c|}{$\begin{array}{l}\text { Prior } \\
\text { CABG } \\
(\%)\end{array}$} & \multicolumn{2}{|c|}{$\begin{array}{c}\text { Prior MI } \\
(\%)\end{array}$} \\
\hline & LES & PES & LES & PES & LES & PES & LES & PES & LES & PES & LES & PES & LES & PES \\
\hline Crimi $^{15)}$ & 73 & 76 & 86 & 79 & 82 & 78 & 27 & 53 & 20 & 34 & 16 & 12 & 34 & 43 \\
\hline Crimi $^{15)}$ & 75 & 76 & 81 & 79 & 86 & 78 & 25 & 53 & 31 & 34 & 20 & 12 & 48 & 43 \\
\hline $\mathrm{Ann}^{9)}$ & 68 & 69 & 48 & 49 & 71 & 71 & 53 & 55 & 11 & 8 & 1 & 2 & 7 & 5 \\
\hline Kitasato $^{23)}$ & 69 & 70 & 81 & 82 & 83 & 82 & 60 & 55 & NA & NA & NA & NA & NA & NA \\
\hline Otsuka $^{16)}$ & 69 & 68 & 72 & 72 & 91 & 94 & 65 & 71 & NA & NA & 12 & 8 & 25 & 25 \\
\hline Otsuka $^{10)}$ & 73 & 73 & 72 & 68 & 85 & 88 & 51 & 50 & 26 & 27 & 5 & 5 & 17 & 16 \\
\hline $\operatorname{Sardi}^{24)}$ & 67 & 67 & 54 & 65 & 99 & 98 & 74 & 63 & 32 & 37 & 32 & 24 & 32 & 40 \\
\hline Tsujita $^{26)}$ & 65 & 67 & 78 & 84 & 76 & 74 & 61 & 76 & 44 & 45 & 10 & 3 & 32 & 34 \\
\hline Yano $^{25)}$ & 64 & 67 & 84 & 87 & 91 & 89 & 75 & 86 & NA & NA & NA & NA & NA & NA \\
\hline Kamoi $^{11)}$ & 65 & 64 & 78 & 70 & 64 & 66 & 48 & 54 & NA & NA & NA & NA & NA & NA \\
\hline Hachinohe ${ }^{17)}$ & 65 & 66 & 67 & 69 & 52 & 55 & 32 & 29 & NA & NA & NA & NA & NA & NA \\
\hline Hachinohe $^{17)}$ & 65 & 66 & 66 & 69 & 52 & 55 & 31 & 29 & NA & NA & NA & NA & NA & NA \\
\hline Simsek $^{12)}$ & NA & NA & NA & NA & NA & NA & NA & NA & NA & NA & NA & NA & NA & NA \\
\hline Ahmed $^{27)}$ & 65 & 67 & 82 & 80 & 52 & 54 & 28 & 30 & NA & NA & NA & NA & NA & NA \\
\hline Ahmed $^{27)}$ & 67 & 67 & 80 & 80 & 53 & 54 & 26 & 30 & NA & NA & NA & NA & NA & NA \\
\hline Ahmed $^{27)}$ & 64 & 67 & 81 & 80 & 54 & 54 & 30 & 30 & NA & NA & NA & NA & NA & NA \\
\hline Higashitani ${ }^{29)}$ & 65 & 64 & 41 & 41 & 43 & 48 & 32 & 41 & 17 & 24 & 12 & 14 & 19 & 21 \\
\hline Otsuka $^{28)}$ & 67 & 66 & 74 & 69 & 84 & 80 & 62 & 59 & NA & NA & 13 & 4 & 35 & 27 \\
\hline Syed $^{31)}$ & 69 & 69 & 59 & 61 & 96 & 95 & 58 & 57 & 33 & 33 & 34 & 30 & 49 & 49 \\
\hline Sukhija ${ }^{30)}$ & 71 & 71 & 53 & 55 & 96 & 92 & 49 & 45 & NA & NA & 21 & 22 & NA & NA \\
\hline Jeong $^{32)}$ & NA & NA & NA & NA & NA & NA & NA & NA & NA & NA & NA & NA & NA & NA \\
\hline
\end{tabular}

LES indicates limus-eluting stent; PES, paclitaxel-eluting stent; MI, myocardial infarction; PCI, percutaneous coronary intervention; CABG, coronary artery bypass grafting; and NA, not applicable.

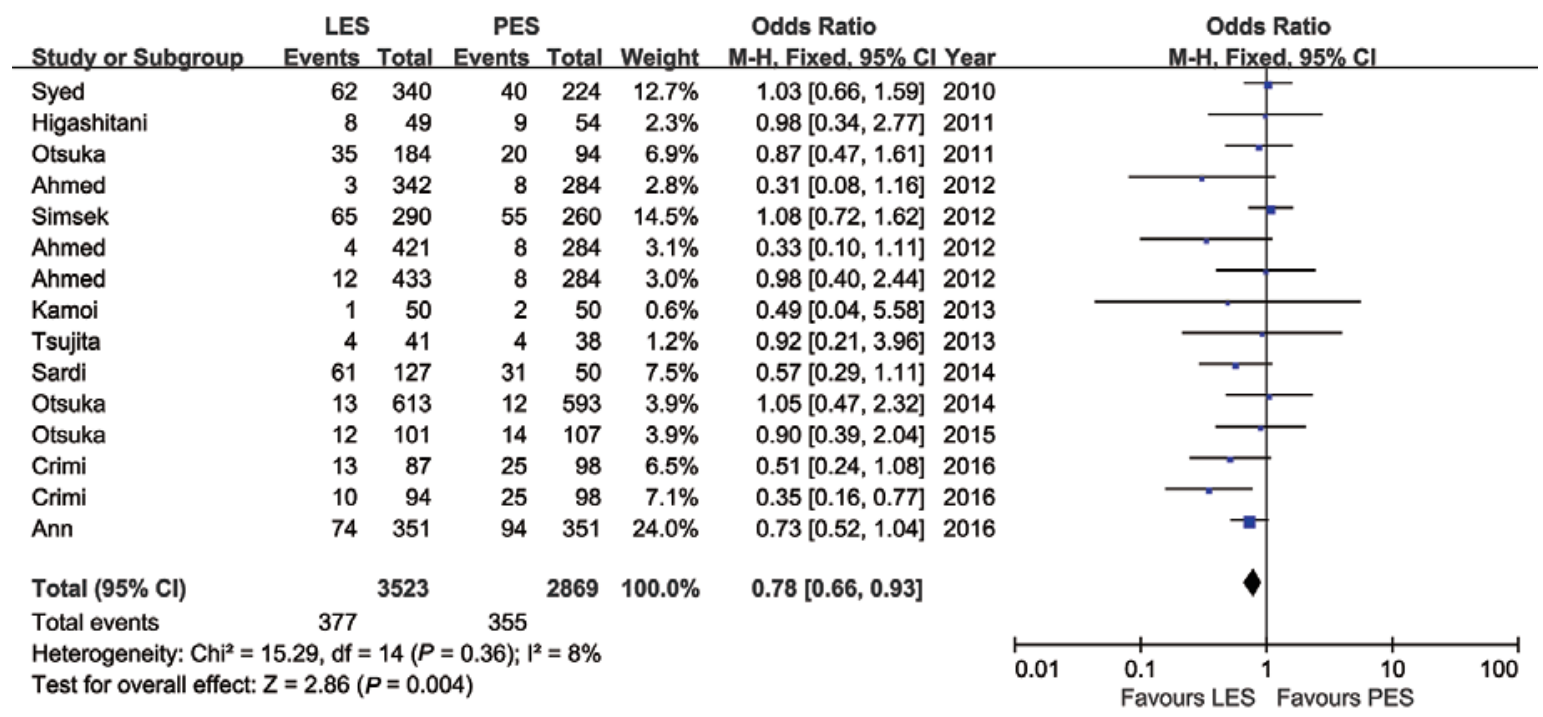

Figure 2. Forest plots for long-term all-cause mortality in CKD patients with LES versus PES.

(15.3\%), no significant difference was found (OR, 0.88; 95\% CI, 0.72-1.07; $P=0.20)$. Significant heterogeneity was observed $\left(I^{2}=65 \%\right)$. Sensitivity analysis did not significantly alter the results. In the subgroup analyses (Table III) by type of coronary stent, only secondary generation DES (SES or ZES) was associated with a significantly lower rate of MACE as compared to PES (OR, 0.61; 95\% CI, 0.38-0.97; $P=0.04)$. The incidence of MACE was comparable in SES and PES cohorts (OR, 1.03; 95\% CI, $0.82-1.28 ; P=0.81$ ) (Figure 3 ).
MI: The incidence of myocardial infarction was comparable in both cohorts. MI was present in $2.8 \%$ and $2.9 \%$ patients in the LES and PES cohorts, respectively (OR, 1.14; 95\% CI, 0.84-1.55; $P=0.41 ; \mathrm{I}^{2}=0 \%$ ) (Figure 3). Neither subgroup analysis by type of coronary stent nor sensitivity analysis significantly altered the results.

TLR and TVR: Seven studies (7,644 patients) reported the number of patients undergoing TLR after coronary stent implantation. TLR was performed in $4.6 \%$ and $4.1 \%$ patients in the LES and PES cohort, respectively. Both co- 
Table III. Subgroup Analysis of Included Studies

\begin{tabular}{|c|c|c|c|c|c|c|c|c|c|c|}
\hline \multirow{2}{*}{ End points } & \multirow{2}{*}{ Studies } & \multicolumn{2}{|c|}{ LES } & \multicolumn{2}{|c|}{ PES } & \multicolumn{3}{|c|}{ Overall effect } & \multicolumn{2}{|c|}{ Heterogeneity } \\
\hline & & Events & Total & Events & Total & OR & $95 \% \mathrm{CI}$ & $P$ value & $\mathrm{I}^{2}$ & $P$ \\
\hline \multicolumn{11}{|l|}{ SES versus PES } \\
\hline Long-term all-cause mortality & 9 & 265 & 2260 & 244 & 1948 & 0.88 & $0.73-1.07$ & 0.21 & 0 & 0.72 \\
\hline Myocardial infarction & 10 & 54 & 2121 & 46 & 1997 & 1.24 & $0.82-1.86$ & 0.31 & 0 & 0.89 \\
\hline Target lesion revascularization & 8 & 139 & 3156 & 105 & 2506 & 1.35 & $0.72-2.54$ & 0.35 & 76 & 0.0001 \\
\hline Target vessel revascularization & 5 & 118 & 1708 & 106 & 1632 & 1.21 & $0.65-2.25$ & 0.54 & 73 & 0.005 \\
\hline MACE & 11 & 680 & 4278 & 604 & 3570 & 1.03 & $0.82-1.28$ & 0.81 & 58 & 0.008 \\
\hline Stent thrombosis & 8 & 33 & 1897 & 18 & 1584 & 1.59 & $0.88-2.85$ & 0.12 & 0 & 0.82 \\
\hline \multicolumn{11}{|l|}{ ZES or EES versus PES } \\
\hline Long-term all-cause mortality & 5 & 51 & 1136 & 80 & 871 & 0.56 & $0.39-0.82$ & 0.003 & 18 & 0.30 \\
\hline Myocardial infarction & 6 & 39 & 1209 & 36 & 945 & 1.14 & $0.70-1.85$ & 0.59 & 0 & 0.43 \\
\hline Target lesion revascularization & 7 & 57 & 1574 & 90 & 2301 & 0.77 & $0.41-1.44$ & 0.42 & 63 & 0.01 \\
\hline Target vessel revascularization & 4 & 30 & 1035 & 26 & 764 & 1.15 & $0.66-1.99$ & 0.62 & 0 & 0.45 \\
\hline MACE & 7 & 160 & 1574 & 321 & 2301 & 0.61 & $0.38-0.97$ & 0.04 & 75 & 0.0004 \\
\hline Stent thrombosis & 5 & 20 & 1136 & 42 & 871 & 0.45 & $0.26-0.77$ & 0.004 & 10 & 0.35 \\
\hline \multicolumn{11}{|l|}{ Dialysis patients } \\
\hline Long-term all-cause mortality & 6 & 121 & 552 & 80 & 393 & 0.78 & $0.54-1.11$ & 0.16 & 0 & 0.92 \\
\hline Myocardial infarction & 6 & 16 & 508 & 8 & 380 & 1.42 & $0.62-3.26$ & 0.41 & 29 & 0.22 \\
\hline Target lesion revascularization & 7 & 122 & 550 & 45 & 416 & 2.23 & $1.53-3.25$ & $<0.001$ & 0 & 0.51 \\
\hline Target vessel revascularization & NA & NA & NA & NA & NA & NA & NA & NA & NA & NA \\
\hline MACE & 5 & 176 & 507 & 89 & 345 & 1.30 & $0.94-1.81$ & 0.11 & 45 & 0.12 \\
\hline Stent thrombosis & 6 & 17 & 542 & 9 & 382 & 1.43 & $0.64-3.18$ & 0.38 & 0 & 0.61 \\
\hline
\end{tabular}

MACE indicates major adverse cardiac events; SES, sirolimus-eluting stent; EES, everolimus-eluting stent; ZES, zotarolimus-eluting stent; LES, limus-eluting stent; PES, paclitaxel-eluting stent; CI, confidence interval; OR, odds ratio; and NA, not applicable, only 1 study reported.

horts were similar (OR, 1.10; 95\% CI, 0.73-1.67; $P=$ $\left.0.65 ; \mathrm{I}^{2}=69 \%\right)$. Moreover, both LES $(6.4 \%)$ and PES (5.6\%) had comparable TVR rates (OR, 1.27; 95\% CI, $0.82-1.67 ; P=1.97 ; \mathrm{I}^{2}=59 \%$ ) (Figure 3 ). Subgroup analysis by type of coronary stent and sensitivity analysis yielded similar results.

Stent thrombosis: Stent thrombosis was reported in 11 studies. Although PES (2.5\%) had a higher stent thrombosis rate as compared to LES $(1.8 \%)$, the difference was not statistically significant (OR, 0.80; 95\% CI, 0.55-1.17; $P=0.25 ; I^{2}=21 \%$ ) (Figure 3 ). Examining first generation and secondary generation DES separately (Table III), we found only secondary generation DES (SES or ZES) had a significantly lower rate of stent thrombosis as compared with PES (OR, 0.45; 95\% CI, 0.26-0.77; $P=0.004 ; \mathrm{I}^{2}=$ $10 \%)$. Stent thrombosis was similar between the SES and PES groups (OR, 1.59; 95\% CI, 0.88-2.85; $P=0.12$ ).

Adverse outcomes in patients on chronic dialysis: $O f$ note, when adverse clinical outcomes were specifically analyzed in patients with chronic dialysis, TLR significantly favored PES as compared to LES (OR, 2.23; 95\% CI, 1.53-3.25; $P<0.001 ; \mathrm{I}^{2}=0 \%$ ) (Table III). No significant differences were found regarding long-term all-cause mortality, MI, TVR, MACE, and ST between LES and PES. A low level of heterogeneity was observed when analyzing the adverse clinical outcomes between LES and PES in patients on chronic dialysis.

Publication bias: There was no indication of a publication bias in the reporting of results on long-term all-cause mortality, either from visualization of the funnel plot (Figure 4$)$ or the Egger weighted regression method ( $P$ value $=0.51$ ).

\section{Discussion}

In the present study, we performed a meta-analysis of 2 randomized trials and 15 cohort studies that had a total of 10,724 participants. The results of the analysis found significantly lower rates of all-cause mortality after LES implantation than after PES implantation. However, LES implantation offered similar MACE, MI, TVR, TLR, and stent thrombosis rates at follow-up compared with PES. Subgroup analyses further revealed that second-generation DES (ZES or EES) rather than first-generation DES (SES) were associated with lower risks of all-cause mortality, MACE and stent thrombosis compared with PES. Of note, in dialysis patients, TLR was significantly higher in LES implantation compared to PES implantation.

Despite the use of new interventional devices, patients with $\mathrm{CKD}$ have higher rates of cardiovascular events including dyslipidemia, increased inflammation, oxidative stress, and adverse clinical outcomes compared with those with normal renal function. ${ }^{33,34)}$ Use of PCI is associated with improved long-term survival when compared with medical therapy alone in patients with severe CKD and acute coronary syndrome. ${ }^{35)}$

Although stent under-expansion, residual reference segment stenosis, and stent fracture after DES implantation have been associated with restenosis and stent thrombosis, ${ }^{36,37)}$ DES implantation has markedly improved the safety and efficacy of percutaneous revascularization. Suzuki and colleagues confirmed the superiority of midterm angiographic outcomes after the placement of EES over SES for de novo coronary stenosis. ${ }^{38)}$ Large randomized trials have shown improved clinical outcomes, such as reduced rates of death and MI, associated with EES 
A mace

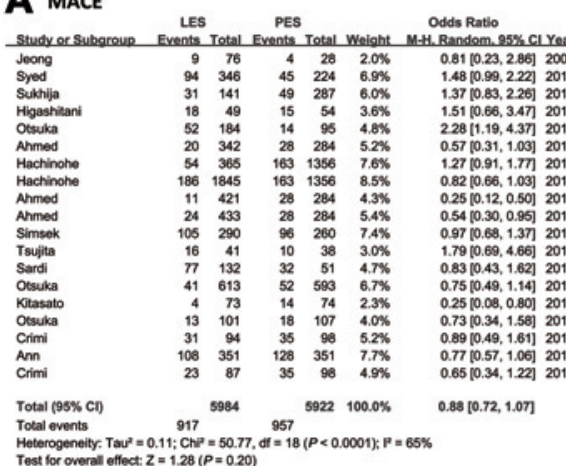

Heterogeneily. Tav" $=0.11 ; C h P=50.77$, df $=18(P<0.0001) ; P=65 \%$
Test for overal effect: $Z=1.28(P=0.20)$

B MI

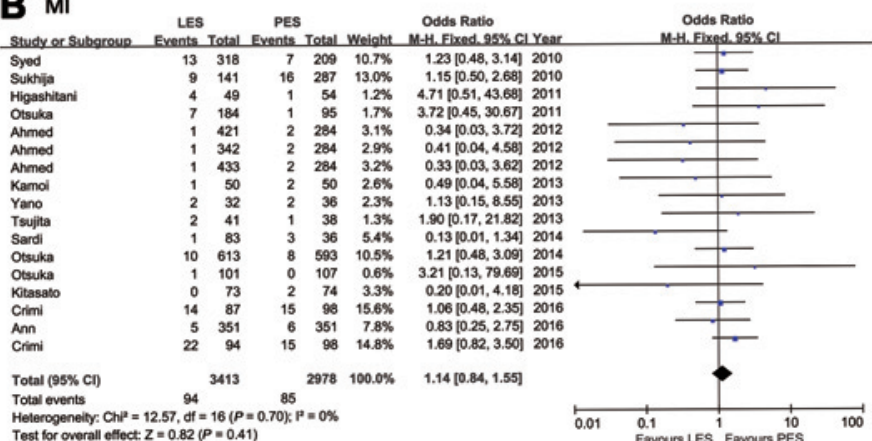

Test for overall effect: $Z=0.82(P=0.41)$

C TVR

LES

PES Odds Ratio

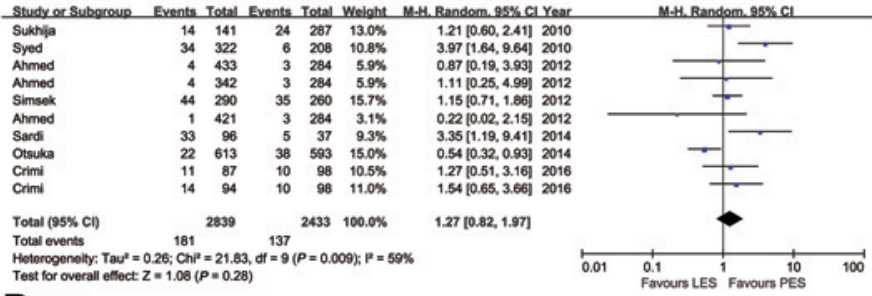

D TLR

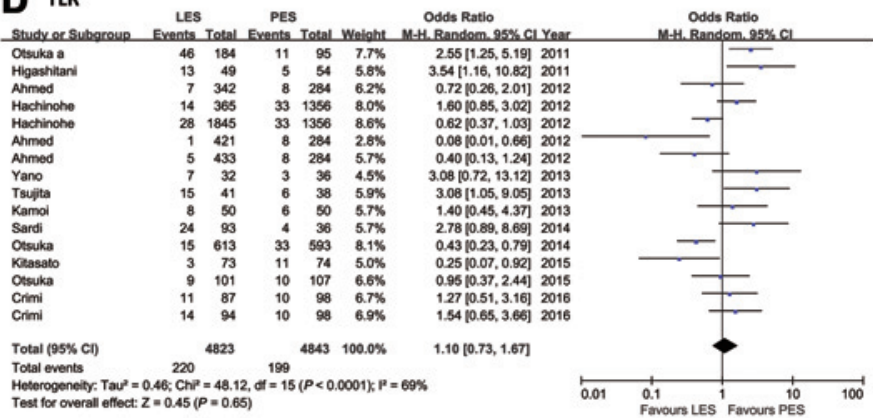

E Stent thrombosis

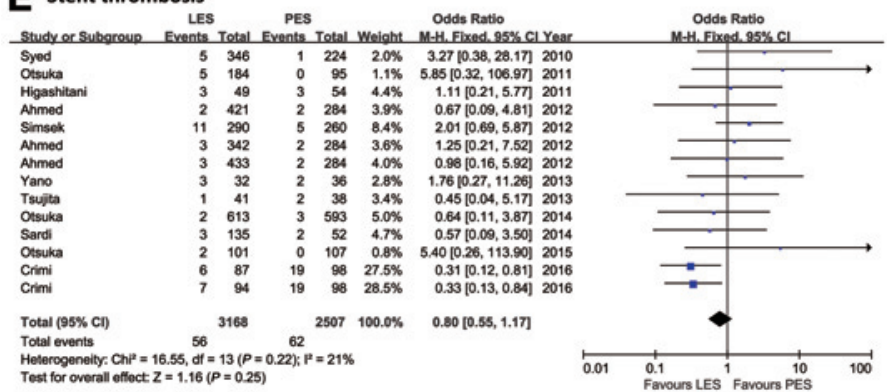

Figure 3. Forest plots for secondary outcomes in CKD patients with LES versus PES, major adverse cardiac events (A); myocardial infarction (B); target vessel revascularization $(\mathbf{C})$; target lesion revascularization $(\mathbf{D})$; stent thrombosis $(\mathbf{E})$. 


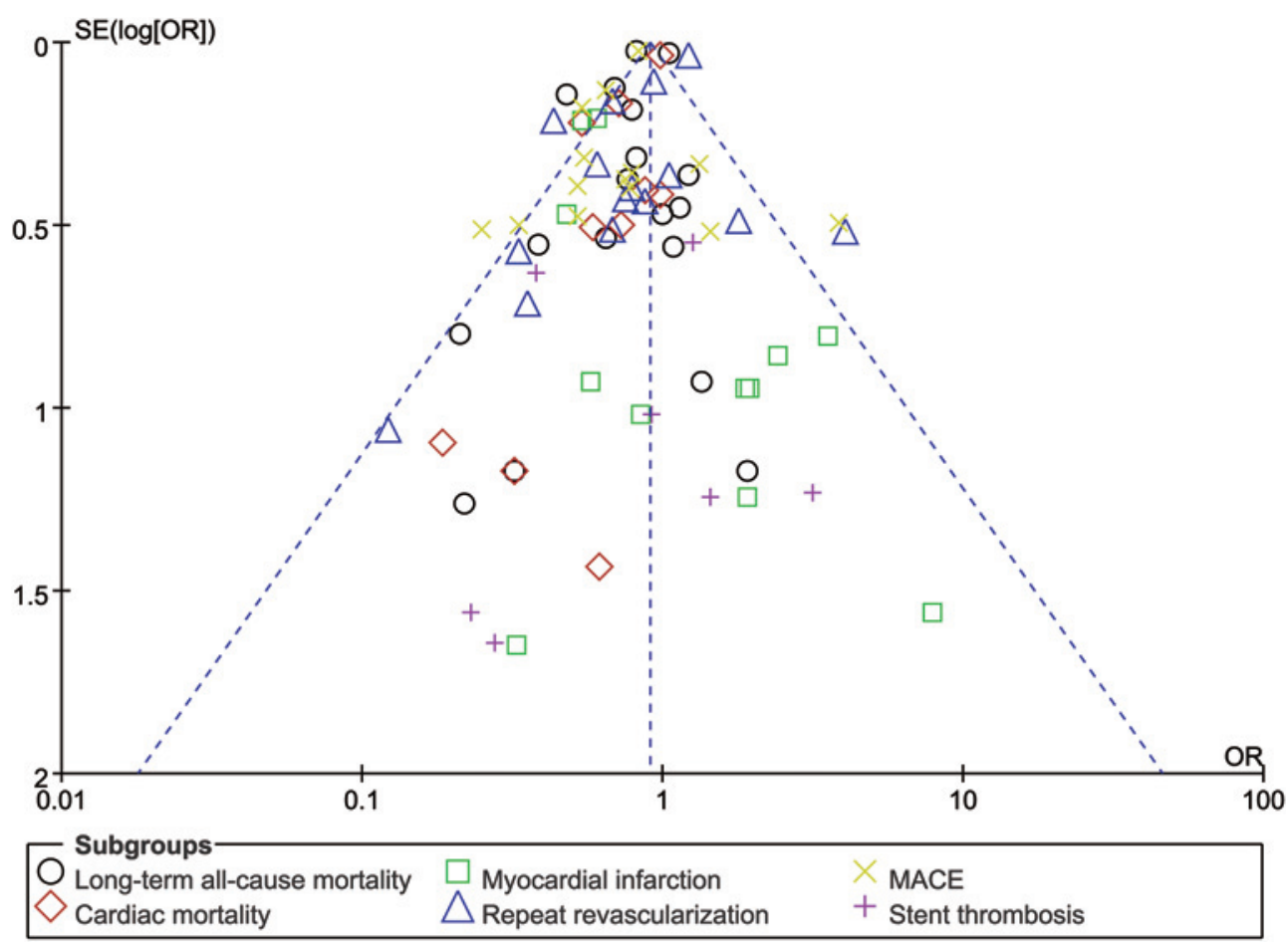

Figure 4. Funnel plot assessing publication bias in the included studies.

and ZES as compared to PES and SES in unselected patients. ${ }^{39-41)}$ By blocking the stimulatory effects of growth factors released after vascular injury and cytokines and inhibiting smooth muscle cell proliferation, EES with its thinner strut design, greater durability, lower risk of strut fracture due to the cobalt chromium platform, lower polymer mass, and limus-based anti-proliferative drugs may induce a lower local inflammatory response, which is associated with a lower incidence of arterial injury and stent thrombosis as compared to first generation DES. ${ }^{23,42)}$ In addition, more rapid endothelialization and preserved endothelial vasomotor response are seen after implantation of the ZES compared with first-generation DES. ${ }^{43)}$

TLR has been demonstrated to be significantly reduced after SES or PES implantation in patients with chronic renal insufficiency compared with bare-metal stent (BMS) implantation. ${ }^{44,45}$ However, when we compared first and second generation DES with PES, our study did not find any significant difference in terms of TLR or TVR in CKD patients. Otsuka and colleagues demonstrated that the PES group was associated with a lower incidence of 1-year MACE compared with the SES group in dialysis patients, ${ }^{28)}$ and also reported that there was no significant difference with regard to MACE between PES and SES implantation in patients with moderate to severe renal insufficiency, and the differences between these reports might have been due to the differences in renal insufficiency severity and study population exclusion criteria. $^{10)}$

Dialysis patients had more complex morphologies such as coronary calcified lesions, and higher incidences of MACE and clinical restenosis after SES implantation, compared to non-dialysis patients. ${ }^{46,47)}$ Notably, our study indicated that PES implantation had lower TLR than LES implantation in dialysis patients. PES, which inhibits instent restenosis and cardiac events in hemodialysis patients, may play an important role in suppression of the chronic inflammatory response in target lesions as compared with SES. ${ }^{25)}$ It is well known that patients on dialysis have more complex coronary lesions, and/or multivessel disease, compared with non-dialysis patients, and are at very high risk of death from coronary artery disease. ${ }^{48,49)}$ It has been shown that sirolimus effects are lacking or minimized in a significant number of HD patients, especially in severely calcified lesions. ${ }^{50)}$ Severely calcified lesions may interfere with diffusion of the more hydrophilic anti-proliferative drugs such as sirolimus, but PES may be less influenced by the adverse lesion conditions than SES as paclitaxel is a hydrophobic potent antineoplastic agent and inhibits leptin. ${ }^{51)}$ Of note, TLR means the stenting site has stenosis progression, while no TLR does not mean the stenting site has no progression. Second generation DES (EES/ZES) have better crossability than PES with an improved platform (stent architecture/alloy/polymer, etc), so we can treat an in-stent restenosis lesion that we cannot treat with PES. This is another possibility for dialysis patients receiving PES to have lower risk of TLR. Thus, whether paclitaxel stents have favorable effects on dialysis patients requires further investigations.

Limitations: Several limitations in the present study must be carefully addressed. First, the pooled results of our review were derived from both randomized controlled trials and retrospective studies. Potential biases such as selec- 
tion bias and treatment bias may exist in the original nonrandomized studies. Second, our analyses of clinical outcomes after second-generation LES implantation combined data for EES and ZES implantation because separate analyses for EES and ZES were subject to limited studies. For example, in dialysis patients, the majority of studies compared SES and PES implantation, and only one study ${ }^{16)}$ included in our meta-analysis provided related data on ZES or EES versus PES in those patients, which prevented us from performing subgroup analysis of second-generation DES versus PES. Third, the outcomes of clinical adverse events could be associated with different stages of CKD, but we only restricted our analysis to dialysis patients. Other stages of CKD could not be stratified and analyzed from the data of the included studies. Fourth, the present study used summarized published event rates for each trial as opposed to individual patient data. Access to individual patient-level data would have enabled further subgroup analyses and propensity analyses to account for any differences in preprocedural risk thereby allowing a better stratification of patients and reduction in potential bias.

All of these limitations may explain the high level of heterogeneity between studies analyzing some adverse outcomes. Notwithstanding the limitations, this study is the first meta-analysis to perform head-to-head comparisons of LES versus PES implantation in patients with CKD as well as patients on chronic dialysis and has shed some light on the optimal stent choice in those patients. Large adequately-powered randomized controlled trials are warranted to confirm the results.

\section{Conclusions}

In patients with CKD, first-generation LES and PES implantation had comparable mortality and morbidity. Second-generation LES implantation was superior to PES in reducing long-term mortality, MACE, and stent thrombosis. However, PES may be more effective than LES in dialysis patients.

\section{Disclosures}

Availability of data and materials: All data and materials used in this research are freely available in electronic databases. References have been provided.

Conflicts of interest: None.

\section{References}

1. Best PJ, Lennon R, Ting HH, et al. The impact of renal insufficiency on clinical outcomes in patients undergoing percutaneous coronary interventions. J Am Coll Cardiol 2002; 39 1113-9.

2. Nakazawa G, Tanabe K, Aoki J, et al. Impact of renal insufficiency on clinical and angiographic outcomes following percutaneous coronary intervention with sirolimus-eluting stents. Catheter Cardiovasc Interv 2007; 69: 808-14.

3. Naidu SS, Selzer F, Jacobs A, et al. Renal insufficiency is an independent predictor of mortality after percutaneous coronary intervention. Am J Cardiol 2003; 92: 1160-4.

4. Murata N, Kaneko H, Yajima J, et al. The prognostic impact of worsening renal function in Japanese patients undergoing percutaneous coronary intervention with acute coronary syndrome. J Cardiol 2015; 66: 326-32.

5. Naidu SS, Selzer F, Jacobs A, et al. Renal insufficiency is an independent predictor of mortality after percutaneous coronary intervention. Am J Cardiol 2003; 92: 1160-4.

6. Yachi S, Tanabe K, Tanimoto S, et al. Clinical and angiographic outcomes following percutaneous coronary intervention with sirolimus-eluting stents versus bare-metal stents in hemodialysis patients. Am J Kidney Dis 2009; 54: 299-306.

7. Shenoy C, Boura J, Orshaw P, Harjai KJ. Drug-eluting stents in patients with chronic kidney disease: A prospective registry study. PLoS One 2010; 5: e15070.

8. Barthelemy O, Helft G, Silvain J, et al. One-year clinical outcomes in patients with chronic renal failure treated by percutaneous coronary intervention with drug-eluting stent. Arch Cardiovasc Dis 2011; 104: 604-10

9. Ann SH, Seo SM, Kim PJ, et al. Sirolimus-eluting stent is superior to paclitaxel-eluting stent for coronary intervention in patients with renal insufficiency: Long-term clinical outcomes. Cardiol J 2016; 23: 637-46.

10. Otsuka M, Yokoi H, Matsuyama Y, et al. Comparison of sirolimus- and paclitaxel-eluting stents in patients with moderate renal insufficiency: results from the J-DESsERT trial. Cardiovasc Revasc Med 2014; 15: 323-8.

11. Kamoi D, Ishii H, Takahashi H, et al. Sirolimus- vs. paclitaxeleluting stent to coronary intervention in dialysis patients. Int $\mathbf{J}$ Cardiol 2013; 165: 533-6.

12. Simsek C, Magro M, Boersma E, et al. Impact of renal insufficiency on safety and efficacy of drug-eluting stents compared to bare-metal stents at 6 years. Catheter Cardiovasc Interv 2012; 80: $18-26$.

13. Hamilos M, Sarma J, Ostojic M, et al. Interference of drugeluting stents with endothelium-dependent coronary vasomotion: evidence for device-specific responses. Circ Cardiovasc Interv 2008; 1: 193-200.

14. Minami Y, Kaneda H, Inoue M, et al. Endothelial dysfunction following drug-eluting stent implantation: a systematic review of the literature. Int J Cardiol 2013; 165: 222-8.

15. Crimi G, Leonardi S, Costa F, et al. Role of stent type and of duration of dual antiplatelet therapy in patients with chronic kidney disease undergoing percutaneous coronary interventions. Is bare metal stent implantation still a justifiable choice? A post-hoc analysis of the all comer PRODIGY trial. Int J Cardiol 2016; 212: 110-7.

16. Otsuka M, Shiode N, Masaoka Y, et al. Comparison of everolimus- and paclitaxel-eluting stents in dialysis patients. Cardiovasc Revasc Med 2015; 16: 208-12.

17. Hachinohe D, Jeong MH, Saito S, et al. Comparison of drugeluting stents in acute myocardial infarction patients with chronic kidney disease. Korean J Intern Med 2012; 27: $397-$ 406.

18. Liberati A, Altman DG, Tetzlaff J, et al. The PRISMA statement for reporting systematic reviews and meta-analyses of studies that evaluate health care interventions: explanation and elaboration. J Clin Epidemiol 2009; 62: e1-34.

19. Jadad AR, Moore RA, Carroll D, et al. Assessing the quality of reports of randomized clinical trials: is blinding necessary? Control Clin Trials 1996; 17: 1-12.

20. Wells GA, Shea B, O'Connell D, et al. The Newcastle-Ottawa scale (NOS) for assessing the quality if nonrandomized studies in meta-analyses. Ottawa: Dept of Epidemiology and Community Medicine, University of Ottawa. Accessed January, 2017. Available at: http://www.ohri.ca/programs/clinical_epidemiology/ oxford.htm.2011.

21. DerSimonian R, Laird N. Meta-analysis in clinical trials. Control Clin Trials 1986; 7: 177-88.

22. Egger M, Davey Smith G, Schneider M, Minder C. Bias in meta-analysis detected by a simple, graphical test. BMJ 1997; 315: 629-34. 
23. Kitasato L, Shimohama T, Ikeda Y, et al. Clinical outcomes of chronic kidney disease patients treated with everolimus-eluting stents (EES) and paclitaxel-eluting stents (PES). Biomed Pharmacother 2015; 72: 6-10.

24. Sardi GL, Loh JP, Torguson R, et al. Drug-eluting stents in patients on chronic hemodialysis: paclitaxel-eluting stents vs. limus-eluting stents. Cardiovasc Revasc Med 2014; 15: 86-91.

25. Yano H, Horinaka S, Yagi H, Ishimitsu T. Comparison of inflammatory response after implantation of sirolimus- and paclitaxel-eluting stents in patients on hemodialysis. Heart Vessels 2013; 28: 308-15.

26. Tsujita H, Hamazaki Y, Nishikura T, et al. Sirolimus-eluting stents versus paclitaxel-eluting stents for coronary intervention in patients with renal failure on hemodialysis. Cardiovasc Interv Ther 2013; 28: 9-15.

27. Ahmed K, Jeong MH, Chakraborty R, et al. Coronary Stents in Patients with ST-Elevation Myocardial Infarction and Chronic Kidney Disease Undergoing Primary Percutaneous Coronary Intervention. Korean Circ J 2012; 42: 830-8.

28. Otsuka M, Toyofuku M, Watanabe N, et al. Clinical usefulness of drug-eluting stents in the treatment of dialysis patients with coronary artery disease. EuroIntervention 2011; 6: 754-9.

29. Higashitani M, Mori F, Yamada N, et al. Efficacy of paclitaxeleluting stent implantation in hemodialysis patients. Heart Vessels 2011; 26: 582-9.

30. Sukhija R, Aronow WS, Palaniswamy C, et al. Major adverse cardiac events in patients with moderate to severe renal insufficiency treated with first-generation drug-eluting stents. Am J Cardiol 2010; 105: 293-6.

31. Syed AI, Ben-Dor I, Collins SD, et al. Sirolimus-eluting stents versus paclitaxel-eluting stents in patients with chronic renal insufficiency. J Interv Cardiol 2010; 23: 33-9.

32. Jeong $\mathrm{YH}$, Hong $\mathrm{MK}$, Lee $\mathrm{CW}$, et al. Impact of significant chronic kidney disease on long-term clinical outcomes after drug-eluting stent versus bare metal stent implantation. Int J Cardiol 2008; 125: 36-40.

33. Schiffrin EL, Lipman ML, Mann JF. Chronic kidney disease: effects on the cardiovascular system. Circulation 2007; 116: 8597.

34. Rubenstein MH, Harrell LC, Sheynberg BV, et al. Are patients with renal failure good candidates for percutaneous coronary revascularization in the new device era. Circulation 2000; 102: 2966-72.

35. Keeley EC, Kadakia R, Soman S, et al. Analysis of long-term survival after revascularization in patients with chronic kidney disease presenting with acute coronary syndromes. Am J Cardiol 2003; 92: 509-14.

36. Uchida Y, Ichimiya S, Ishii $\mathrm{H}$, et al. Impact of coronary stent fracture on restenotic neointimal tissue characterization after drug-eluting stent implantation. Int Heart J 2017; 58: 861-7.

37. Oba Y, Hoshide S, Mitama T, et al. Recurrent early coronary stent thrombosis under chronic disseminated intravascular coagulation. Int Heart J 2017; 58: 988-92.

38. Suzuki K, Ishikawa $\mathrm{T}$, Mutoh $\mathrm{M}$, et al. Propensity-score matched comparison of midterm angiographic outcomes of sirolimus- versus everolimus- and biolimus-eluting stents for de novo coronary stenosis. Int Heart J 2017; 58: 320-7.
39. Jensen LO, Thayssen P, Hansen HS, et al. Randomized comparison of everolimus-eluting and sirolimus-eluting stents in patients treated with percutaneous coronary intervention: the Scandinavian Organization for Randomized Trials with Clinical Outcome IV (SORT OUT IV). Circulation 2012; 125: 1246-55.

40. Camenzind E, Wijns W, Mauri L, et al. Stent thrombosis and major clinical events at 3 years after zotarolimus-eluting or sirolimus-eluting coronary stent implantation: a randomised, multicentre, open-label, controlled trial. Lancet 2012; 380: 1396-405.

41. Leon MB, Nikolsky E, Cutlip DE, et al. Improved late clinical safety with zotarolimus-eluting stents compared with paclitaxeleluting stents in patients with de novo coronary lesions: 3-year follow-up from the ENDEAVOR IV (Randomized Comparison of Zotarolimus- and Paclitaxel-Eluting Stents in Patients With Coronary Artery Disease) trial. JACC Cardiovasc Interv 2010; 3: $1043-50$.

42. Doostzadeh J, Clark LN, Bezenek S, et al. Recent progress in percutaneous coronary intervention: evolution of the drugeluting stents, focus on the XIENCEV drug-eluting stent. Coron Artery Dis 2010; 21: 46-56.

43. Hamilos M, Sarma J, Ostojic M, et al. Interference of drugeluting stents with endothelium-dependent coronary vasomotion: evidence for device-specific responses. Circ Cardiovasc Interv 2008; 1: 193-200.

44. Okura H, Nakamura M, Kotani J, Kozuma K. Gender-specific outcome after paclitaxel-eluting stent implantation in Japanese patients with coronary artery disease - sub-analysis of the Japan TAXUS Express2 post-marketing survey. Circ J 2013; 77: 14305.

45. Kuchulakanti PK, Torguson R, Chu WW, et al. Impact of chronic renal insufficiency on clinical outcomes in patients undergoing percutaneous coronary intervention with sirolimuseluting stents versus bare metal stents. Am J Cardiol 2006; 97: 792-7.

46. Ota T, Umeda H, Yokota S, et al. Relationship between severity of renal impairment and 2-year outcomes after sirolimus-eluting stent implantation. Am Heart J 2009; 158: 92-8.

47. Jung HH, Kim SW, Han H. Inflammation, mineral metabolism and progressive coronary artery calcification in patients on haemodialysis. Nephrol Dial Transplant 2006; 21: 1915-20.

48. Herzog CA, Ma JZ, Collins AJ. Comparative survival of dialysis patients in the United States after coronary angioplasty, coronary artery stenting, and coronary artery bypass surgery and impact of diabetes. Circulation 2002; 106: 2207-11.

49. Naidu SS, Selzer F, Jacobs A, et al. Renal insufficiency is an independent predictor of mortality after percutaneous coronary intervention. Am J Cardiol 2003; 92: 1160-4.

50. Ikari Y, Tanabe K, Koyama Y, et al. Sirolimus eluting coronary stent implantation in patients on maintenance hemodialysis: the OUCH study (outcome of cypher stent inhemodialysis patients). Circ J 2012; 76: 1856-63.

51. Nakazawa G, Tanabe K, Aoki J, et al. Impact of renal insufficiency on clinical and angiographic outcomes following percutaneous coronary intervention with sirolimus-eluting stents. Catheter Cardiovasc Interv 2007; 69: 808-14. 